\title{
riccafd
}

Revista Iberoamericana de Ciencias de la Actividad Física y el Deporte

\section{LESIONES EN JÓVENES GIMNASTAS FEMENINAS DE ACROBÁTICA DE LA ÉLITE NACIONAL}

\section{Injuries in young acrobatic female gymnasts of the national elite}

Mercedes Vernetta ${ }^{1}$, Isabel Montosa², Jesús López-Bedoya1

\begin{abstract}
${ }^{1}$ Mercedes Vernetta. Doctora en Educación Física y Profesora Titular de Universidad. Departamento de Educación Física y Deporte. Facultad de Ciencias de la Actividad Física y el Deporte. Universidad de Granada, España. E-mail: vernetta@ugr.es

${ }^{2}$ Isabel Montosa. Licenciada en Ciencias de la Actividad Física y el deporte. Departamento de Educación Física y Deporte. Facultad de Ciencias de la Actividad Física y el Deporte. Universidad de Granada, España. E-mail: isabelmontosa@gmail.com

3 Jesús López-Bedoya. Doctor en Educación Física y Profesor Titular de Universidad. Departamento de Educación Física y Deporte. Facultad de Ciencias de la Actividad Física y el Deporte. Universidad de Granada, España E-mail:jlopezb@ugr.es
\end{abstract}

\section{AGRADECIMIENTOS}

A todas las gimnastas y entrenadores por la colaboración en el estudio.

Código UNESCO: 3212 Salud Pública

Clasificación Consejo de Europa: 17. Otras: Actividad Física y Salud.

Correspondencia:

Isabel Montosa

isabelmontosa@gmail.com

DOI:http://dx.doi.org/10.24310/riccafd.2018.v7i3.5542

\section{RESUMEN}

La gimnasia acrobática está asociada inevitablemente a la aparición de posibles lesiones. El objetivo fue analizar su incidencia a gimnastas de nivel competitivo. Se realizó un estudio prospectivo, transversal y descriptivo a través de un cuestionario donde participaron 40 jóvenes entre 12 y 18 años. Se analizó las lesiones sufridas y las características del entrenamiento. Dichas lesiones se produjeron más en los entrenamientos (95,5\%) que en competición. El 57,77\% fueron localizadas en la extremidad inferior, $28,88 \%$ en el tronco $13,33 \%$ en la extremidad superior. Los sitios anatómicos más afectados fueron el tobillo y espalda (33,33\% y $28,9 \%$, respectivamente). En cuanto al tipo de lesión destacan los esguinces (40\%) y contracturas $(26,7 \%)$. La mayoría de las lesiones ocurrieron en la parte técnica, durante los movimientos grupales estáticos y dinámicos. El estudio demuestra que existe un predominio de lesiones ligamentosas y musculares, siendo los miembros inferiores la zona más afectada. 
Palabras clave: Gimnasia acrobática, lesiones, incidencia.

\begin{abstract}
ABTRACT
Acrobatic gymnastics is inevitably associated with the appearance of possible injuries. The objective was to analyze its incidence to gymnasts of competitive level. A prospective, cross-sectional and descriptive study was carried out through a questionnaire where 40 young people between 12 and 18 years old participated. The injuries suffered and the characteristics of the training were analyzed. These injuries occurred more in training (95.5\%) than in competition. $57.77 \%$ were located in the lower extremity, $28.88 \%$ in the trunk $13.33 \%$ in the upper extremity. The most affected anatomical sites were the ankle and back (33.33\% and $28.9 \%$, respectively). Regarding the type of injury, sprains $(40 \%)$ and contractures $(26.7 \%)$ stand out. Most of the injuries occurred in the technical part, during the static and dynamic group movements. The study shows that there is a predominance of ligamentous and muscular injuries, with the lower limbs being the most affected area.
\end{abstract}

Keyword: Acrobatic gymnastics, injuries, incidence.

\title{
INTRODUCCIÓN
}

La Gimnasia Acrobática (GA) es una disciplina gimnástica relativamente moderna, con cuatro décadas de historia desde su inclusión en 1973 en la Federación Internacional de Deportes Acrobáticos. Es el último deporte gimnástico integrado en la Federación Internacional de Gimnasia (FIG) en 1999. En España, es uno de los deportes gimnásticos más novedosos, comenzando su andadura en el ámbito competitivo a nivel nacional e internacional en el 2004.

Se trata de un deporte cooperativo por excelencia definido como "deporte Sociomotriz, donde existe siempre la presencia de uno o varios compañeros que sincronizan sus acciones motrices en un espacio estable reglado, para conseguir la realización de figuras o pirámides humanas ${ }^{(1)}$. Por tanto, es un deporte de situaciones de relación casi constantes a través de un contacto corporal y ayuda mutua, donde los acróbatas realizan unas habilidades motrices específicas establecidas de antemano, en busca de una gran perfección técnica y coreográfica. Para las habilidades de grupo, los gimnastas actúan como " bases o portores "y " ágiles o tops " en las modalidades de pareja y portores, ágiles y "medios" en las modalidades de grupos: tríos femenino y cuartetos masculinos ${ }^{(2)}$.

Por razones evidentes en función del perfil motor de esta disciplina, las gimnastas más pequeñas y jóvenes actuán como ágiles ocupando la parte superior en las figuras y pirámides colectivas ${ }^{(3)}$, realizando elementos de flexibilidad, 
equilibrio y combinaciones encima del portor o, grandes saltos acrobáticos en fase aérea mediante propulsiones de los portores para recepcionar de nuevo sobre ellos o en el suelo. De ahí, que en función del puesto o rol desempeñado por el gimnasta sus características morfológicas son específicas y diferentes ${ }^{(4)}$.

La gran exigencia técnica junto al alto nivel deportivo demanda en estas gimnastas la constante inclusión de dificultades de gran valor en el Código de Puntuación aumentando así su riesgo a lesionarse. Además, muchos de los elementos técnicos exigidos requieren hipermovilidad de la columna vertebral, la cadera y el hombro, por ello, aproximadamente el $50 \%$ de las gimnastas acrobáticas reportan dolor resultante de cambios degenerativos en la cadera, rodilla y columna vertebral|(5).

Al ser la última modalidad gimnástica incluida en la FIG, existe poca documentación y sobre todo falta de estudios epidemiológicos relacionados con este deporte $^{(3)(5)}$ como ocurre en la gimnasia aeróbica frente a otros deportes gimnásticos más veteranos y olímpicos como la gimnasia artística masculina y femenina o la gimnasia rítmica(6).

De ahí, la necesidad de evaluar las lesiones en la práctica de este deporte y observar detalladamente diferentes aspectos relacionados con el entrenamiento, con el fin de obtener una información válida que sirva de base para la prevención de las mismas. A este respecto, Kolt y Kirkby ${ }^{(7)}$, indican que el registro de las lesiones asociadas con sus factores causales, pueden ser un valioso método profiláctico y ejercer influencia en el rendimiento.

Por lo tanto, debido a la falta de conocimiento de trabajos que hayan analizado las lesiones en esta población española, el objetivo de este estudio fue conocer la frecuencia, los patrones y la severidad de las lesiones producidas durante la práctica en gimnastas jóvenes de esta modalidad deportiva.

\section{MATERIAL Y MÉTODO}

Estudio descriptivo, transversal a 40 gimnastas femeninas (45\% ágiles y $55 \%$ portoras).Todas de nivel nacional con éxitos competitivos y muchos de ellas, un $81 \%$ han formando parte de la selección española a nivel internacional. En general las gimnastas tenían una experiencia media de entrenamiento superior a cuatro años, $(4,92 \pm 3,62)$ realizaban una media de tres a cinco sesiones de entrenamiento por semana $(3,2 \pm 1,01)$, entre 3 y 4 horas/día. El número de competiciones por año era entre 5 y 7 por año. Los criterios de inclusión fueron: gimnastas federadas, participantes en campeonatos nacionales, de sexo femenino, con edades entre 10 y 18 años, modalidad: parejas femeninas y tríos que quisieran participar en el estudio y al ser menor de edad contar con el consentimiento de sus padres y entrenadores. Las características de la muestra se pueden observar en la Tabla 1. 
Tabla 1: Características antropométricas y edad.

\begin{tabular}{llll}
\hline & Ágiles & Portoras & TOTAL \\
\hline $\mathrm{N}$ & 18 & 22 & 40 \\
Edad (años) & $12,94( \pm 2,07)$ & $15,91( \pm 1,71)$ & $14,62( \pm 2,35)$ \\
Masa corporal $(\mathrm{Kg})$ & $35,10( \pm 4,55)$ & $53,4( \pm 7,52)$ & $45,17( \pm 11,16)$ \\
Talla $(\mathrm{m})$ & $1,47( \pm 0,08)$ & $1,61( \pm 0,05)$ & $1,72( \pm 1,55)$ \\
IMC $^{*}$ & $16,02( \pm 1,32)$ & $20,54( \pm 2,23)$ & $18,50( \pm 2,95)$ \\
\hline
\end{tabular}

Los datos se muestran en valores promedio \pm desviación estándar

*IMC (Índice de masa corporal)

En la firma del consentimiento informado, se destacaba el carácter voluntario de su participación, así como la confidencialidad de los datos del cuestionario en cumplimiento de la Declaración de Helsinki.

Para la recogida de la información se utilizó un cuestionario retrospectivo validado en estudios precedentes en Gimnasia Aeróbica( ${ }^{(8)}$, y aplicados en Gimnasia Rítmica $^{(9)}$. Se trata de un cuestionario que permite conocer, las características de los participantes (sexo, edad, modalidad, categoría competitiva y valores antropométricos: peso, talla); las características del entrenamiento (tiempo de práctica: horas y días de entrenamiento, utilización de pista reglamentaria y material de seguridad utilizados habitualmente); lesiones sufridas durante su carrera deportiva (número, tipo o naturaleza, localización, gravedad, momento, mecanismo, tratamiento y secuelas). El formato del cuestionario se conservó en su totalidad y sólo hubo modificaciones en la sesión de entrenamiento en la parte de la técnica específica para reflejar el perfil motor de esta disciplina gimnástica.

En la valoración de la gravedad de las lesiones se diferenció: lesiones leves (al menos interrumpen un día de entrenamiento), moderadas (obliga al gimnasta a interrumpir entre 8 y 21 días sus entrenamientos y requieren tratamiento), graves (supone de 22 días en adelante de baja deportiva, a veces hospitalización o incapacidad permanente ${ }^{(10)}$. Sobre el tipo de lesión se identificaron lesiones musculares, articulares, ligamentosas, óseas y heridas ${ }^{(11)}$.

Se calculó la incidencia lesional a través de la fórmula: número de lesiones / horas de exposición x 1000 horas, de manera general para los entrenamientos y competiciones ${ }^{(3,9,12)}$. Igualmente, con el peso y la talla se calculó el Índice de Masa Corporal (IMC) referido al índice de Quetelet $(\mathrm{Kg} / \mathrm{m} 2)$.

\section{Análisis Estadístico}

Se realizó un análisis descriptivo para todas las variables. Los datos se muestran en frecuencias observadas y porcentaje, salvo en las variables cuantitativas, en cuyo caso se estimaron como valores promedio y desviación estándar.

Para conocer las diferencias entre los dos grupos determinados por el rol competitivo (ágil y portora), se utilizó las pruebas $t$ de Student para la comparación 
de medias de muestras independientes, en función de la normalidad en la distribución de la muestra. En las variables cualitativas expresadas en porcentajes se ha aplicado el test de Chi-cuadrado. El criterio de significación se estableció en $p$ $<0,05$. Todos los datos fueron analizados usando el programa informático "Statistical Package for the Social Sciences" (SPSS) v. 22.0 (SPSS Inc., Chicago, IL).

\section{RESULTADOS}

Entre las 40 gimnastas entrevistadas, 29 reportaron alguna lesión. La Tabla 2 muestra el número total de lesiones según los diferentes roles. El tiempo total de exposición fue de 44.413 horas, con un total de 45 lesiones, de las cuales el $95,55 \%(f=43)$ se produjeron durante el entrenamiento y $4,44 \%(f=2)$ en competición, de forma que el porcentaje de lesiones fue significativamente mayor $(p<0.01)$ durante el entrenamiento. Contrastando el número de lesiones con la cantidad total de horas de exposición se obtuvo una incidencia lesional de 1,01 por cada $1.000 \mathrm{~h}$ de exposición.

Tabla 2. Número de lesiones y momento de la lesión y modalidad competitiva.

\begin{tabular}{|c|c|c|c|c|}
\hline \multicolumn{5}{|c|}{ ¿Has tenido alguna lesión en Gimnasia Acrobática? } \\
\hline & $\begin{array}{l}\text { Ágiles } \\
f \%\end{array}$ & $\begin{array}{l}\text { Portoras } \\
f \%\end{array}$ & $\begin{array}{l}\text { Total } \\
f \%\end{array}$ & p-valor \\
\hline $\mathrm{Si}$ & $12(66,7 \%)$ & $17(77,3 \%)$ & $29(72,5 \%)$ & คด००* \\
\hline No & $6(33,3 \%)$ & $5(22,7 \%)$ & $11(27,5 \%)$ & \\
\hline \multicolumn{5}{|c|}{ Momento de la lesión } \\
\hline & $\begin{array}{l}\text { Ágiles } \\
f \%\end{array}$ & $\begin{array}{l}\text { Portoras } \\
f \%\end{array}$ & $\begin{array}{l}\text { Total } \\
f \%\end{array}$ & \\
\hline Entrenamiento & 16 (100\%) & $27(93,1 \%)$ & $43(95,5 \%)$ & \\
\hline Competición & 0 & $2(6,89 \%)$ & $2(4,4 \%)$ & 0,335 \\
\hline \multicolumn{5}{|c|}{ Modalidad competitiva } \\
\hline & $\begin{array}{l}\text { Ágiles } \\
f \%\end{array}$ & $\begin{array}{l}\text { Portoras } \\
f \%\end{array}$ & $\begin{array}{l}\text { Total } \\
f \%\end{array}$ & \\
\hline Pareja femenina & $6(37,5)$ & $15(51,72)$ & $21(46,66)$ & \\
\hline Trio femenino & $10(62,5)$ & $14(48,27)$ & $24(53,55)$ & $0,000 *$ \\
\hline
\end{tabular}

*nivel de significación $p<0,05$

En cuanto al tipo de lesión, estructura corporal, localización y severidad de las mismas, según rol, los datos se muestran en la Tabla 3. Existe un claro predominio de las lesiones en los miembros inferiores, siendo los esguinces los más frecuentes, predominando en función de la cantidad de días de incapacidad 
funcional, las de tipo leve (44,4\%). Por otro lado, se puede observar que ambas (portoras y ágiles) se lesionan más en el tobillo y en la espalda. No se encontraron diferencias significativas entre ambos grupos en ninguna de las variables analizadas.

Tabla 3: Tipo de lesiones, estructura corporal, localización y severidad de las mismas por rol.

\begin{tabular}{|c|c|c|c|}
\hline Tipo de lesiones & $\begin{array}{l}\text { Ágiles } \\
f \%\end{array}$ & $\begin{array}{l}\text { Portoras } \\
f \%\end{array}$ & $\begin{array}{l}\text { Total } \\
f \%\end{array}$ \\
\hline Contracturas & $6(37,5)$ & $6(20,7)$ & $12(26,7)$ \\
\hline Esguinces & $9(56,3)$ & $9(31)$ & $18(40)$ \\
\hline Fisuras y fracturas & $1(6,3)$ & $6(20,7)$ & $7(15,55)$ \\
\hline Tendinitis & 0 & $6(20,7)$ & $6(13,33)$ \\
\hline Otras & 0 & $2(6,9)$ & $2(4,4)$ \\
\hline Total & 16 & 29 & 45 \\
\hline Estructura Corporal & $f \%$ & $f \%$ & $f \%$ \\
\hline Miembro Superior & $1(6,25)$ & $5(17,31)$ & $6(13,33)$ \\
\hline Tronco & $6(37,5)$ & $7(24,13)$ & $13(28,88)$ \\
\hline Miembro Inferior & $9(56,25)$ & $17(58,62)$ & $26(57,77)$ \\
\hline Total & 16 & 29 & 45 \\
\hline Localización & $f \%$ & $f \%$ & $f \%$ \\
\hline Rodilla & 0 & $4(13,8)$ & $4(8,9)$ \\
\hline Tobillo & $8(50)$ & $7(24,1)$ & $15(33,3)$ \\
\hline Espalda & $6(37,5)$ & $7(24,1)$ & $13(28,9)$ \\
\hline Muslo & $1(6,3)$ & $2(10,3)$ & $3(6,66)$ \\
\hline Muñeca & $1(6,3)$ & $3(10,3)$ & $4(8,9)$ \\
\hline Pie & 0 & $4(13,8)$ & $4(8,9)$ \\
\hline Hombro & 0 & $2(6,9)$ & $2(4,4)$ \\
\hline Severidad & $f \%$ & $f \%$ & $f \%$ \\
\hline Leves & $7(43,8)$ & $13(44,8)$ & $20(44,4)$ \\
\hline Moderadas & $5(31,1)$ & $12(41,3)$ & $17(37,8)$ \\
\hline Graves & $4(25)$ & $4(13,7)$ & $8(17,8)$ \\
\hline Total & 16 & 29 & 45 \\
\hline
\end{tabular}

Atendiendo al momento de producción dentro de la sesión de entrenamiento, la mayor carga lesional se concentra en la técnica específica, sobre todo en los elementos grupales con un $41,86 \%$ (23,2\% dinámicos y $18,60 \%$ estáticos) seguido del los elementos individuales 32,55 \% (18,6\% estáticos y 13,9\% dinámicos), no existiendo diferencias significativas entre las ágiles y portoras (Tabla 4). El mecanismo lesional más frecuente fue la sobrecarga, con un $32,55 \%$ del total de las lesiones reportadas, seguido del mal apoyo con $(30,23 \%)$. 
Tabla 4: Momento de la lesión sesión de entrenamiento

\begin{tabular}{llll}
\hline Técnica específica & $\begin{array}{l}\text { Ágiles } \\
\boldsymbol{f} \%\end{array}$ & $\begin{array}{l}\text { Portoras } \\
\boldsymbol{f} \%\end{array}$ & $\begin{array}{l}\text { Total } \\
\boldsymbol{f} \%\end{array}$ \\
\hline Grupal estático & $2(12,5)$ & $6(22,2)$ & $8(18,60)$ \\
Grupal dinámico & $3(18,7)$ & $7(25,9)$ & $10(23,2)$ \\
Individual estático & $3(18,7)$ & $5(18,5)$ & $8(18,6)$ \\
Individual dinámico & $3(18,7)$ & $3(11,1)$ & $6(13,9)$ \\
Calentamiento & $2(12,5)$ & $3(11,1)$ & $5(11,6)$ \\
Coreografía & $1(6,25)$ & $1(3,7)$ & $2(4,6)$ \\
Preparación física & $2(12,5)$ & $2(7,4)$ & $4(9,30)$ \\
\hline
\end{tabular}

\section{DISCUSIÓN}

Este estudio tuvo como objetivo principal describir las lesiones sufridas en jóvenes gimnastas de acrobática de la élite nacional, atendiendo al número, tipo, áreas potenciales, momento de producirse y gravedad de la misma teniendo en cuenta la cantidad de días de competición y sesiones de entrenamiento. Pocos estudios han analizado la incidencia lesional en gimnastas jóvenes de esta disciplina gimnástica, de ahí la importancia de los resultados obtenidos para futuras investigaciones.

Los resultados mostraron que las lesiones durante los entrenamientos son mucho más numerosas que en competiciones con diferencias significativas estadísticamente, datos coincidentes con los estudios de gimnasia acrobática(3). Esta misma tendencia se da en otras disciplinas gimnásticas ${ }^{(8,9)}$, que muestran mayor porcentaje de lesiones durantes los entrenamientos y escasa en competiciones. El mayor porcentaje de lesiones se ubica en las portoras $(f=29)$ frente a las ágiles $(f=16)$ con diferencias estadísticamente significativas entre roles.

Se debe de tener en cuenta, que las portoras tienen más edad y más años de experiencia en horas y días de entrenamiento con las ágiles, lo que podría explicar ese mayor número de lesiones.

De los datos se desprende que es fundamental dosificar la carga de entrenamiento para optimizar la eficacia de los mismos ${ }^{(13)}$, ya que cargas abusivas de trabajo podrían aumentar el riesgo de lesión. Y sobre todo, en gimnastas jóvenes de 11-15, que tienen mayor vulnerabilidad a lesionarse si el volumen de entrenamiento está por encima de un cierto umbral, por encontrarse en un periodo crítico de crecimiento acelerado de la adolescencia(3).

La tasa de lesiones fue de 1,01 por cada $1000 \mathrm{~h}$ de exposición sumando competiciones y entrenamiento, siendo inferiores a los 2,94 reportados en gimnasia acrobática ${ }^{(3)}$; y muy similar a los obtenidos por Cupisiti el al. ${ }^{(14)}$ con 1.08 y ligeramente inferior a los de Vernetta et at. ${ }^{(9)}$ con 1,46, ambos con gimnastas de rítmica. 
Con respecto al IMC medio fue de $16,02 \mathrm{~kg} / \mathrm{m}^{2}$ para las ágiles, estando la mayoría de las gimnastas en normopeso o peso ligeramente bajo, "Delgadez grado I" según los valores de Cole, et al. ${ }^{(15)}$, resultados similares a las parejas femeninas 16,76 y grupos femeninos 16,13 de esta disciplina obtenidos por Taboada et al. ${ }^{(16)}$, estableciéndose igualmente similitudes con las gimnastas de rítmica. ${ }^{(9,17)}$, con valores de $16.12 \mathrm{~kg} / \mathrm{m}^{2}$ y $16.82 \mathrm{~kg} / \mathrm{m}^{2}$ respectivamente, pero más pequeño a los de Ávila-Carvalho, et al. ${ }^{(18)}$, con $18,75 \mathrm{Kg} / \mathrm{m}^{2}$.

En cuanto a las portoras, presentaron un IMC de $20,54 \mathrm{~kg} / \mathrm{m}^{2}$ estando todas en normopeso y con valores similares a las portoras de grupo y pareja femeninas. ${ }^{(16)}$, con $19,84 \mathrm{~kg} / \mathrm{m}^{2}$ y $21,09 \mathrm{~kg} / \mathrm{m}^{2}$ respectivamente. Se hallaron diferencias significativas en cuanto al IMC al comparar ágiles con portoras $(p<0,01)$, lo cual muestra la evolución natural del aumento del IMC conforme se incrementa la edad en este periodo, ya que las ágiles son menores que las portoras ${ }^{(19)}$

Por otro lado, en función del tipo de lesiones fueron las ligamentosas y tendinosas las más frecuentes, datos que van en la línea de los resultados hallados por otros trabajos de acrobática ${ }^{(3,20)}$. Purnell et al, ${ }^{(3)}$ señalan que los esguinces ligamentosos o tendinopatía de Aquiles en los tobillos, la tendinopatía de rodilla, la disfunción patelofemoral, y esguinces de ligamento / cápsula en las muñecas son las más corrientes, similar a las acróbatas analizados en nuestro estudio.

En cuanto a la severidad de las lesiones fue de tipo leve $(44,4 \%)$ y moderado $(37,8 \%)$, lo que supuso una recuperación de no más de una semana o menos de un mes, por lo que la inactividad no repercutió del todo en el rendimiento deportivo, ya que los gimnastas podían seguir con un entrenamiento modificado relacionado fundamentalmente con su condición física específica, evitando utilizar la zona lesionada. En el estudio de Purnell et al. ${ }^{(3)}$, la mayoría de las lesiones de los acróbatas $(66,7 \%)$ requirieron menos de 11 días de descanso del entrenamiento, datos similares a los obtenidos en nuestra muestra. Igualmente Caine y Nassar ${ }^{(21)}$, afirman que el $41 \%$ de las lesiones que se producen en gimnasia requieren menos de 8 días de recuperación (lesiones leves) y el 33\% entre 8 y 21 días. En general, las necesidades de rehabilitación se basaron en una recuperación activa y temprana, haciendo uso del frío, vendaje, reposo y masaje para acelerarla.

Atendiendo a la estructura corporal, se corroboran los resultados obtenidos en gimnasia acrobática. ${ }^{(3,20,22)}$, siendo los miembros inferiores $(48,88 \%)$ la zona más dañada. Estos datos igualmente, son coincidentes con los estudios en gimnastas de artística $^{(21,23) ;}$ de rítmicas $\left({ }^{7,9,14)}\right.$ y de aerobic ${ }^{(8)}$.

Generalmente, los estudios con gimnastas de diferentes modalidades acrobáticas de niveles nacionales e internacionales reflejan que la mayor carga lesional se produce en las extremidades inferiores, siendo los ligamentos y tejidos blandos los que tienen un predominante porcentaje de daños ${ }^{(24)}$. En $\mathrm{GA}^{(3,20)}$ igualmente, las lesiones ligamentosos fueron las más frecuentes en los miembros inferiores. 
La segunda y tercera zona más lesionada de la muestra fueron el tronco y el miembro superior con $(26,66 \%$ y $24,4 \%$ respectivamente), datos que contrasta con los de Purnell et al. ${ }^{(3)}$, siendo la extremidad superior la segunda zona más afectada $(24,1 \%)$ y por último, la columna vertebral con $(17,9 \%)$ y se aleja de los de Rego et al. ${ }^{(22)}$, donde el mayor número de lesiones en practicantes de acrobática fue en los miembros superiores con un $(34,7 \%)$. En cuanto al sitio anatómico, destacar el tobillo como la zona más predominante, datos similares a los obtenidos en gimnastas de acrobática $^{(3)}$ y en gimnasia artística donde la incidencia de lesiones en el tobillo y rodilla fue superior en los ejercicios de suelo, especialmente durante la fase de aterrizaje debido al impacto sobre estas articulaciones ${ }^{(25-27)}$.

Además de los esguinces de tobillo, el dolor lumbar de los acróbatas de nuestro estudio es un problema frecuente, resultados que concuerdan con los obtenidos en los practicantes de acrobática(3) y rítmica $^{(9)}$ ya que son disciplinas que requieren muchas posiciones diferentes con niveles extremos de flexión o extensión de la columna.

Sería por tanto recomendable, proteger la espalda con algún cinturón lumbar, así como incluir dentro de las sesiones de entrenamiento, un trabajo preventivo adicional que incluyan ejercicios de reforzamiento abdominal y programas de educación postural para mejorar la estabilización de esta zona ${ }^{(9,28)}$.

Por otro lado, la tercera zona afectada en nuestro estudio fueron las extremidades superiores, principalmente las muñecas como ocurre en la gimnasia

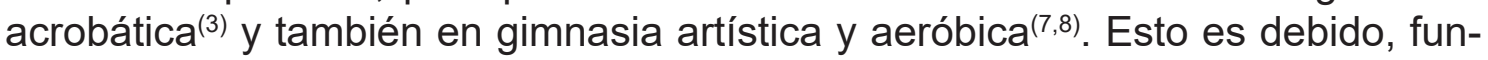
damentalmente por la sobrecarga que sufren los gimnastas sobre dicha articulación al realizar determinados elementos de dificultad que soportan grandes cargas como el peso de su cuerpo sobre las mismas ${ }^{(8,22,23)}$, o el peso del ágil en los ejercicios grupales en acrobática ${ }^{(3,20,22)}$.

En cuanto a la sesión de entrenamiento, fue durante la fase técnica $(68,88 \%$, sumando elementos grupales e individuales tanto estáticos como dinámicos) cuando se produjo el porcentaje de lesiones más elevado, al igual que sucede en otros estudios de esta especialidad ${ }^{(3)}$. Datos en consonancia con la gimnasia aeróbica, el ballet clásico y contemporáneo, donde el porcentaje de lesión es el más alto durante la realización de elementos técnicos. Sin embargo es contrario a los resultados hallados en gimnasia rítmica con jóvenes gimnastas siendo el porcentaje más elevado durante el calentamiento ${ }^{(9)}$.

Dentro de la parte técnica, los elementos de mayor incidencia lesiva fueron los grupales tanto en ágiles como portores. En los acróbatas que desempeñan el rol de ágiles fundamentalmente se producen en las recepciones de las habilidades dinámicas donde son lanzados por sus portoras a alturas elevadas para realizar elementos de rotaciones en diferentes ejes de gran complejidad de dificultad. La mayoría de las lesiones se produjeron en el tobillo debido a fallos en los aterrizajes de elementos por mal apoyo con un $(30,23 \%)$, resultados que concuerdan 
con otros estudios de gimnastas de artística, donde se considera la recepción o aterrizaje de los gimnastas a su llegada al suelo la fase más propensa de lesiones $^{(6,29)}$.

En las portoras, se constata los datos obtenidos en parejas femeninas ${ }^{(3)}$, donde gran incidencia de lesiones se dan a nivel muscular por uso excesivo, en las habilidades grupales estáticas, ya que estas gimnastas deben de mantener posturas sostenidas mientras soportan el peso de su pareja. Estos datos son coincidentes con deportes afines como cheerleading ${ }^{(30)}$, en cuanto a que integran rutinas de ejercicios con música que implican elevaciones, pirámides y lanzamientos, con soporte musical, donde igualmente, fueron las elevaciones y los montajes de sus compañeros soportados sobre sus manos las habilidades que conllevó más lesiones con un 34\%.

Destacar que estos resultados puedan tener relación con el gran tiempo invertido en los portores en las posiciones de base para mantener a sus ágiles en las posiciones de equilibrio, donde los porcentaje de fuerza estática son elevados en la realización de sus acciones motrices durante la ejecución de sus ejercicios de competición ${ }^{(31)}$. Además, la perfección técnica que exige el Código de Puntuación en estos elementos demandan un número elevado de repeticiones durante el entrenamiento, lo que conlleva un sobreuso o fatiga muscular, siendo estos uno de los mecanismos de mayor incidencia lesional con el $32,55 \%$ en nuestra muestra.

De ahí, la importancia de un mayor control en este tipo de movimiento, insistiendo en un aprendizaje progresivo con tareas adecuadas hasta llegar a una técnica correcta y un control en la carga (volumen e intensidad) de los mismos durante el entrenamiento ${ }^{(1)}$. Estas medidas preventivas, deberían de considerarse como imprescindibles ${ }^{(32)}$, ya que muchas de las lesiones que empiezan siendo aguda por un simple dolor de espalda, se pueden convertir fácilmente en crónicas sobre todo en portores de la modalidad de parejas, quienes en el estudio de Purnell et al. ${ }^{(3)}$, el $(52,9 \%)$ de todas las lesiones fueron crónicas.

Finalmente resaltar, que todas nuestras gimnastas del estudio hacían uso de la pista reglamentaria, siendo los materiales de seguridad y protección más utilizados los vendajes $(40,88 \%)$ y tobilleras y muñequeras (26\%). A veces su uso se limitaba a período de recuperación de la lesión y otras, como medida preventiva ante posible recidiva.

Las limitaciones en nuestra investigación han sido por un lado, el diseño transversal, que proporciona evidencias por asociación, pero no relaciones causales, así que de cara al futuro, sería interesante realizar estudios longitudinales. Por otro lado, los datos obtenidos del cuestionario están basados en informes realizados por las propias gimnastas, por lo que podrían tener cierto nivel de sesgo a pesar de que el procedimiento y registro utilizado ha mostrado su eficacia en otros deportes gimnásticos ${ }^{(8-10)}$. No obstante, sería más aconsejable disponer de especialistas en medicina que registren las lesiones de cara a obtener datos más 
precisos. Finalmente, no tener una muestra más grande y heterogénea en cuanto al número de gimnastas en esta disciplina que abarque la totalidad existente en España y las diferentes modalidades competitivas (parejas mixtas y grupos masculinos).

De ahí la necesidad de aumentar la producción científica en esta área de estudio planteando nuevos retos de investigación con diferentes líneas. No obstante, este trabajo como primer estudio en España, proporciona información relevante de la epidemiología en este deporte. Nuestra intención es seguir ampliando el estudio en próximas temporadas limando dichas limitaciones para obtener datos concluyentes y centrarnos posteriormente en futuras investigaciones que abarquen programas de prevención con diferentes estrategias de intervención para reducir los posibles factores de riesgo de lesiones en esta disciplina tan nueva y eminente en nuestro país.

\section{CONCLUSIONES}

Según los resultados obtenidos la región corporal más afectada fue los miembros inferiores, predominando las lesiones ligamentosos (esguinces), seguida de la espalda (contracturas y distensiones). La fase de la sesión de mayor porcentaje de lesiones fue en el entrenamiento técnico, siendo los elementos grupales específicos dinámicos y estáticos. La mayoría de las lesiones fueron leves y moderadas.

A raíz de los resultados, de cara al futuro, se debería de optimizar los programas de prevención realizando en los entrenamientos ejercicios de pre-activación muscular en todas las posibles situaciones de aterrizaje ${ }^{(33)}$, ya que esta fase parece ser crítica ${ }^{(26,27)}$. Además si se tiene en cuenta que el tipo de lesión más prevalente fue el esguince, específicamente en tobillo, se debería de implementar en la planificación del entrenamiento, un trabajo preventivo adicional que incluyan tareas o ejercicios propioceptivos ${ }^{(34)}$.

Igualmente, sería crucial realizar cargas progresivas ya que en gimnasia acrobática los portores soportan mucho peso sobre su columna aumentando el riesgo de lesión, además, los ágiles realizan movimientos que pueden producir contusiones por lo que es importante planificar el entrenamiento respetando la edad de las gimnastas, buscando siempre su bienestar y salud.

Finalmente, consideramos relevante como indica Oliveira ${ }^{(35)}$, promover una intervención multidisciplinar por parte de todos los profesionales (entrenadores, médicos, fisioterapeutas) para que las jóvenes gimnastas puedan practicar un mejor deporte en un contexto de mayor placer y seguridad. 


\section{BIBLIOGRAFÍA}

1. Vernetta M, López Bedoya J, Gutiérrez A. La Creatividad en la Gimnasia Acrobática. En Martínez A, Día, P (Comp). Creatividad y Deporte. 2008; 133-54.

2. Federación Internacional de Gimnasia. Código de Puntuación Gimnasia Acrobática, 2017-2020. Madrid: RFEG

3. Purnell M, Shirley D, Nicholson I, Adams R. Acrobatic gymnastics injury: occurrence, site and training risk factors. Phys Ther Sport. 2010.;11:40-6.

4. Taboada-Iglesias Y, Gutiérrez-Sánchez Á, Vernetta M. Índices de Proporcionalidad y Composición Corporal de la Élite de Gimnasia Acrobática. Int. J. Morphol. 2015; 33(3), 996-1001.

5. Anwajler J, Wojna D, Stepak A, Skolimowski T. The influence of sports acrobatic training on the range of mobility of the spine and the upper and lower extremities. Fizjoterapia Polska. 2005; 5, 57-64.

6. Abalo R, Gutiérrez A, Vernetta M. Aerobic Gymnastic Injuries. Review article. Rev int med cienc act fís deporte. 2013; 13(49), 183-98.

7. Kolt GS, Kirkby RJ. Epidemiology of injury in elite and subelite female gymnasts: a comparison of retrospective and prospective findings. Br J Sports Med. 1999; 33(4): 312-18.

8. Abalo R, Vernetta M, Gutiérrez A. Analysis of the lesional incidence in the spanish gymnastics elite aerobic competition. Rev Bras Med Esporte. 2013;19(5):375-8.

9. Vernetta M, Montosa I, López Bedoya J. Análisis de las lesiones deportivas en jóvenes practicantes de gimnasia rítmica de competición en la categoría infantil. Rev Andal Med Deporte. 2016;9(3):105-9.

10. Marsall SW, Marshall SW, Covassin T, Dick R, Lawrence GN, Agel J. Descriptive epidemiology of collegiate women's gymnastics injuries: National Collegiate Athletic Association Injury Surveillance System, 1988 - 1989 through 2003 - 2004. J Athl Train. 2007;42(2):234-40.

11. Egocheaga J, Urraca J, Del Valle M, Rozada A. Estudio epidemiológico de las lesiones en el rugby. Arch Med Deporte. 2003;20(93):22-6.

12. Dowdell T. Is gymnastics a dangerous sport in the Australian Club context?. Sci Gymnastics J. 2011;3(2):13-25.

13. Jespersen E, Verhagen E, Holst R, Klakk H, Heidemann M, Rexen CT, Franz C, Wedderkopp N. Total body fat percentage and body mass index and the association with lower extremity injuries in children: a 2.5-year longitudinal study. Br J Sports Med. 2014; 48:1497-1502.

14. Cupisti A. Injury survey in competitive sub-elite rhythmic gymnasts: results from a prospective controlled study. J Sports Med Phys Fitness. 2007;47:203-7. 
15. Cole TJ, Flegal KM, Nicholls D, Jackson AA. Body mass index cut off sto define thinness in children and adolescents. International survey. 2007; 335:194-97.

16. Taboada-Iglesias Y, Gutiérrez-Sánchez A, Vernetta M. Anthropometric profile of elite acrobatic gymnasts and prediction of role performance. J Sports Med Phys Fit. 2015; 33(3): 996-1001.

17. Poliszczuk T, Broda D, Poliszczuk D. Changes in somatic parameters and dynamic balance in female rhythmic gymnasts over a space of two years. Pol J Sport Tourism. 2012; 19:240-45.

18. Ávila-Carvalho L, Klentrou P, Luz Palomero M, Lebre E. Body composition profile of elite group rhythmic gymnasts. Sci Gymnastics J. 2012;2(4):21-32.

19. Garagorri JM. Hipercrecimientos: sistema diagnóstico. An Pediatr. 2004; 60(4), 291-95.

20. Grapton X, Lion A, Gauchard GC, Barrault D, Perrin PP. Specific injuries induced by the practice of trampoline, tumbling and acrobatic gymnastics. Knee Surg Sports Traumatol Arthrosc. 2013; 21:494-99.

21. Caine D, Nassar L. Gymnastics injuries. Med Sport Sci. 2005; 48: 18-58.

22. Rego F, Reis M, Oliveira R. Lesões em Ginastas Portugueses de Competição das Modalidades de Trampolins, Ginástica Acrobática, Ginástica Artística e Ginástica Rítmica na Época 2005/2006. Rev Portuguesa de Fisioterapia no Desporto, 2007; 21 (1): 21-7.

23. Marshall SW, Marshall SW, Covassin T, Dick R, Lawrence GN, Agel J. Descriptive epidemiology of collegiate women's gymnastics injuries: National Collegiate Athletic Association Injury Surveillance System, 1988 - 1989 through 2003 - 2004. J Athl Train. 2007;42(2):234-40.

24. Meeusen R, Duclos M, Foster C, Fry A, Gleeson M, Nieman D, Raglin J, Rietjens G, Steinacker J, Urhausen A. (2013). Prevention, diagnosis and treatment of the overtraining syndrome: Joint consensus statement of the European College of Sport Science (ECSS) and the American College of Sports Medicine (ACSM). Eur. J. Sport Sci, 13 (1): 1-24.

25. Kirialanis P, Malliou P, Beneka A, Giannakopoulos K. Occurrence of acute lower limb injuries in artistic gymnasts in relation to event and exercise phase. $\mathrm{Br} \mathrm{J}$ Sports Med. 2003;37:137-9.

26. Harringe $M L$, Renströn $P$, Werner $S$. Injury incidence, mechanism and diagnosis in top-level teamgym: a prospective study conducted over one Seaton. Scand J Med Sci Sports. 2007;17, 115-19.

27. O'Kane JW, Levy MR, Pietila KE, Caine DJ, Schiff MA. Survey of injuries in Seattle area levels 4 to 10 female club gymnasts. Clin J Sport Med. 2011;21(6):486-92. 
28. Vidal J, Borrás PA, Ortega FB, Cantallops J, Ponseti X, Palou, P. Effects of Postural Education on Daily Habits in Children. Int J Sports Med. 2011;32(4),303-8.

29. Nunomura M, Pires RF, Carrara P. Análise do treinamento da ginástica artística brasileira. Rev Bras Cienc Esporte. 2009;31:25-40.

30. Shields BJ, Smith GA. Epidemiology of strain/sprain injuries among cheerleaders in the United States. Am J Emerg Med. 2011;29(9):1003-12.

31. Vernetta M, Jiménez J, López-Bedoya J. La utilización del registro de los tiempos de intervención de las acciones motrices en la gimnasia acrobática. 2007. En Lecturas Educación Física y Deportiva. Revista Digital. 110. www.efdeportes.com. Buenos Aires.

32. Daly R, Bass S, Finch C. Balancing the risk of injury to gymnasts: How wffective are the counter measures?. Br J Sports Med. 2001; 35, 8-19.

33. Chassé M, Fergusson DA, Chen Y. Body mass index and the risk of injury in adults: a cross-sectional study. Int J Obesity. 2014; 38: 1403-9.

34. Rodríguez-Camacho DF, Correa-Mesa JF, Camargo-Rojas DA, Correa- Morales JC. Prevalencia de lesiones en gimnastas pertenecientes a la Liga de Gimnasia de Bogotá, D.C. Rev. Fac. Med. 2016;64:S85-91.

35. Oliveira R. Lesões nos Jovens Atletas: conhecimento dos fatores de risco para melhor prevenir. Rev Portuguesa de Fisioterapia no Desporto, 2007; 3(1), 33-8.

Referencias totales citadas: 35

Referencias citadas correspondientes a la Rev Ib CC Act Fis Dep: 0 